\title{
Transcription factor NF-YA promotes a malignant phenotype by upregulating fatty acid synthase expression
}

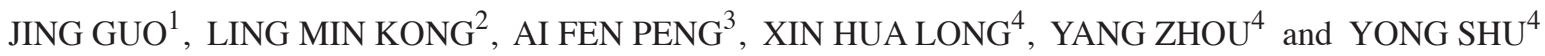 \\ ${ }^{1}$ Department of Oral and Maxillofacial Surgery, The Affiliated Stomatological Hospital of Nanchang University, Nanchang, \\ Jiangxi 330006; ${ }^{2}$ Department of Orthopedics, The Central People's Hospital of Ji'an City, Ji'an, Jiangxi 343000; \\ ${ }^{3}$ Jiangxi University of Traditional Chinese Medicine, Nanchang, Jiangxi 330004; ${ }^{4}$ Department of Orthopedics, \\ The First Affiliated Hospital of Nanchang University, Nanchang, Jiangxi 330006, P.R. China
}

Received October 15, 2015; Accepted September 14, 2016

DOI: $10.3892 / \mathrm{mmr} .2016 .5897$

\begin{abstract}
Recent studies have revealed that increased expression of the alpha subunit of nuclear transcription factor $\mathrm{Y}$ (NF-YA) is associated with the malignant phenotype of various tumors. However, whether elevated expression of NF-YA promotes a malignant phenotype in osteosarcoma (OS), and the molecular mechanisms underlying this predicted effect is currently unknown. In the present study, small hairpin RNA (shRNA)-mediated knockdown of endogenous NF-YA significantly inhibited the migration and invasion capabilities of OS cells in vitro, whereas ectopic expression of NF-YA increased the migration and invasion capabilities of these cells. In addition, the induction of upregulated NF-YA expression on the malignant phenotype of OS cells was attenuated by silencing fatty acid synthase (FASN) expression. Furthermore, the expression level of FASN was increased by upregulating NF-YA, while decreased FASN expression was observed following NF-YA silencing in OS cells. The results of the present study suggest that NF-YA may promote a malignant phenotype in OS cells, in part, by activating the FASN signaling pathway, which may represent a promising target for the management of OS.
\end{abstract}

\section{Introduction}

Osteosarcoma (OS) is the most common primary malignant bone tumor in children and adolescents, and demonstrates a high tendency to metastasize (1). The estimated worldwide incidence rate is 4,000,000 cases/year (2). Although the 5-year survival rates of patients with primary OS has been significantly improved from $30-50 \%$ to $60-90 \%$ by combining surgery with multiagent chemotherapy (3-5), the efficacy of chemotherapy is

Correspondence to: Professor Yong Shu, Department of Orthopedics, The First Affiliated Hospital of Nanchang University, 17 Yong Wai Zheng Street, Nanchang, Jiangxi 330006, P.R. China E-mail: shuyong1957@126.com

Key words: alpha subunit of nuclear transcription factor Y, fatty acid synthase, osteosarcoma, malignant phenotype reduced by the development of drug resistance (6). It is estimated that $<30 \%$ of patients with recurrent disease achieve 5-year survival (7-9). Therefore, the identification of novel molecular targets and signaling pathways is necessary to improve the effectiveness of chemotherapy for the management of OS.

Nuclear transcription factor $\mathrm{Y}(\mathrm{NF}-\mathrm{Y})$ is a ubiquitous protein, composed of three subunits; NF-YA, NF-YB and NF-YC. The genes that encode these proteins are highly conserved from yeast to mammals, with NF-YA acting as the regulatory subunit of the trimer (10). It has been demonstrated that NF-Y regulates gene expression by binding to promoter regions, and NF-Y is essential for cell proliferation (11-13). Multiple studies have recently demonstrated that increased expression of NF-Y in various cancers is associated with poor prognosis (14-16). However, whether elevated expression of NF-Y promotes a malignant phenotype in OS cells, and the molecular mechanisms underlying these putative effects remains unknown.

Fatty acid synthase (FASN) is an important enzyme involved in energy metabolism. Our previous studies suggested that FASN is overexpressed in OS cells and promoted its malignant phenotype $(17,18)$. However, the mechanism underlying FASN overexpression remains unclear.

The present study aimed to determine whether NF-YA is involved in mediating the malignant phenotype of OS via regulation of FASN expression, by investigating the malignant phenotype of OS cells and FASN expression following knockdown or overexpression of NF-YA.

\section{Materials and methods}

Cell lines. The human OS cell lines, U2-OS and HOS, and the human osteoblast cell line, HOB, were purchased from the Cell Bank of Type Culture Collection of the Chinese Academy of Sciences (Shanghai, China). The U2-OS and HOS cells were cultured in Ham's/F-12 medium (Gibco; Thermo Fisher Scientific, Inc., Waltham, MA, USA), and HOB cells were cultured in Dulbecco's modified Eagle medium (Gibco; Thermo Fisher Scientific, Inc.), both supplemented with $10 \%$ fetal bovine serum (FBS; Gibco; Thermo Fisher Scientific, Inc.), 100 U/ml penicillin and $100 \mathrm{U} / \mathrm{ml}$ streptomycin. Cells were cultured at $37^{\circ} \mathrm{C}$ in $5 \% \mathrm{CO}_{2}$. 
Table I. Primer information.

Primer (product size)

Sequence (5'-3')

FASN (262 bp)

Forward: GTCGGAGAACTTGCAGGAGT

$\beta$-actin (295 bp)

Reverse: TCCTCGGAGTGAATCTGGGT

NF-YA (238 bp)

Forward: TCACCCACACTGTGCCATCATCGA

Reverse:CAGCGGAACCGCTCATTGCCAATGG

Forward: TTGTTGGTCAGGGTTTACAGC

Reverse: ACGCTCCACGATGTCACTAA

FASN, fatty acid synthase; NF-YA, alpha subunit of nuclear transcription factor Y.

$R N A$ isolation and reverse transcription-quantitative polymerase chain reaction ( $R T-q P C R)$. Total RNA from OS and HOB cells was extracted using TRIzol ${ }^{\circledR}$ (Invitrogen; Thermo Fisher Scientific, Inc.) according to the manufacturer's protocol. Total RNA concentration was determined by measuring absorbance at a wavelength of $260 \mathrm{~nm}$ and purity was determined by calculating the $260 / 280 \mathrm{~nm}$ ratio with a BioPhotometer (Eppendorf, Hamburg, Germany). The mRNA expression levels of NF-YA and FASN were evaluated by RT-qPCR, using $\beta$-actin as the internal reference gene. The Two-Step RT kit (Promega Corporation, Madison, WI, USA) was used according to the manufacturer's protocol to synthesize cDNA, which was used as the template for amplification. TaqMan ${ }^{\circledR}$ Real-Time PCR Master Mixes (Thermo Fisher Scientific, Inc.) were used for amplification under the following cycling conditions: An initial denaturation step at $95^{\circ} \mathrm{C}$ for $1 \mathrm{~min}$, followed by 40 cycles of denaturation at $95^{\circ} \mathrm{C}$ for $15 \mathrm{sec}$, annealing at $58^{\circ} \mathrm{C}$ for $20 \mathrm{sec}$ and extension at $72^{\circ} \mathrm{C}$ for $20 \mathrm{sec}$. Data was normalized using the $2^{-\Delta \Delta C q}$ method (19). All procedures were performed according to the manufacturer's protocols, with primer sequences as listed in Table I. A total of six independent experiments were performed over multiple days.

Lentivirus vector construction and cell transfection. HEK293 cells were used to produce lentiviral vectors, with the viral titer of $2 \times 10^{8}$. To construct vectors for upregulating and downregulating NF-YA and FASN expression, the wild-type sequences and reverse complementary sequences (5'-ACTGAC TGACCAAACAGCAATAGTTCGACAGAGCACAGGACA

CAAGGCCTGTTAC-3') were inserted into lentivirus vectors (Biomiga Inc., San Diego, CA, USA). U2-OS and HOS cells were transfected using polybrene (Genomeditech, Shanghai, China) with lentivirus vectors (multiplicity of infection=20) to upregulate NF-YA (Lv-up-NF-YA), downregulate NF-YA (Lv-down-NF-YA) and downregulate FASN (Lv-down-FASN). A non-targeting lentivirus vector ( $\mathrm{Lv}$-neg) was used as a negative control. The transfection efficiency was evaluated by fluorescence microscopy, as the lentiviral vectors expressed green fluorescent protein.

Western blot analysis. Total protein from cells was extracted using radioimmunoprecipitation assay lysis buffer (Invitrogen; Thermo Fisher Scientific, Inc.) containing $60 \mu \mathrm{g} / \mathrm{ml}$ phenylmethylsulfonyl fluoride, according to the manufacturer's protocol. Protein concentration was determined using the Bradford assay.
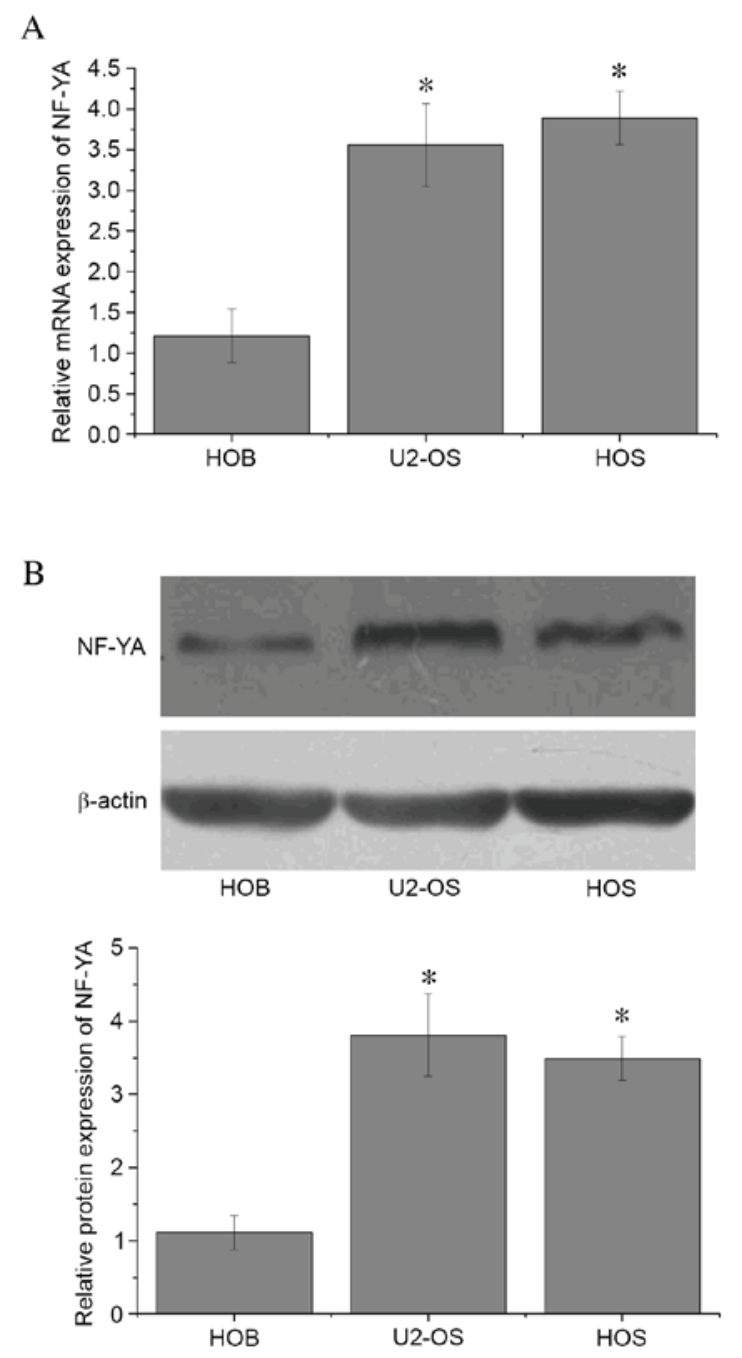

Figure 1. NF-YA mRNA and protein expression levels in human osteoblast HOB cells, and human osteosarcoma U2-OS and HOS cells. (A) mRNA expression levels were detected by reverse transcription-quantitative polymerase chain reaction relative to $\beta$-actin. (B) Protein expression levels were detected by western blot and quantified relative to $\beta$-actin. Data are presented as the mean \pm standard deviation of six independent experiments. ${ }^{*} \mathrm{P}<0.05$ vs. HOB. NF-YA, alpha subunit of nuclear transcription factor Y.

Proteins $(10 \mu \mathrm{g})$ were separated by SDS-PAGE and transferred to polyvinylidene difluoride membranes. The membranes were probed with the following primary antibodies overnight 

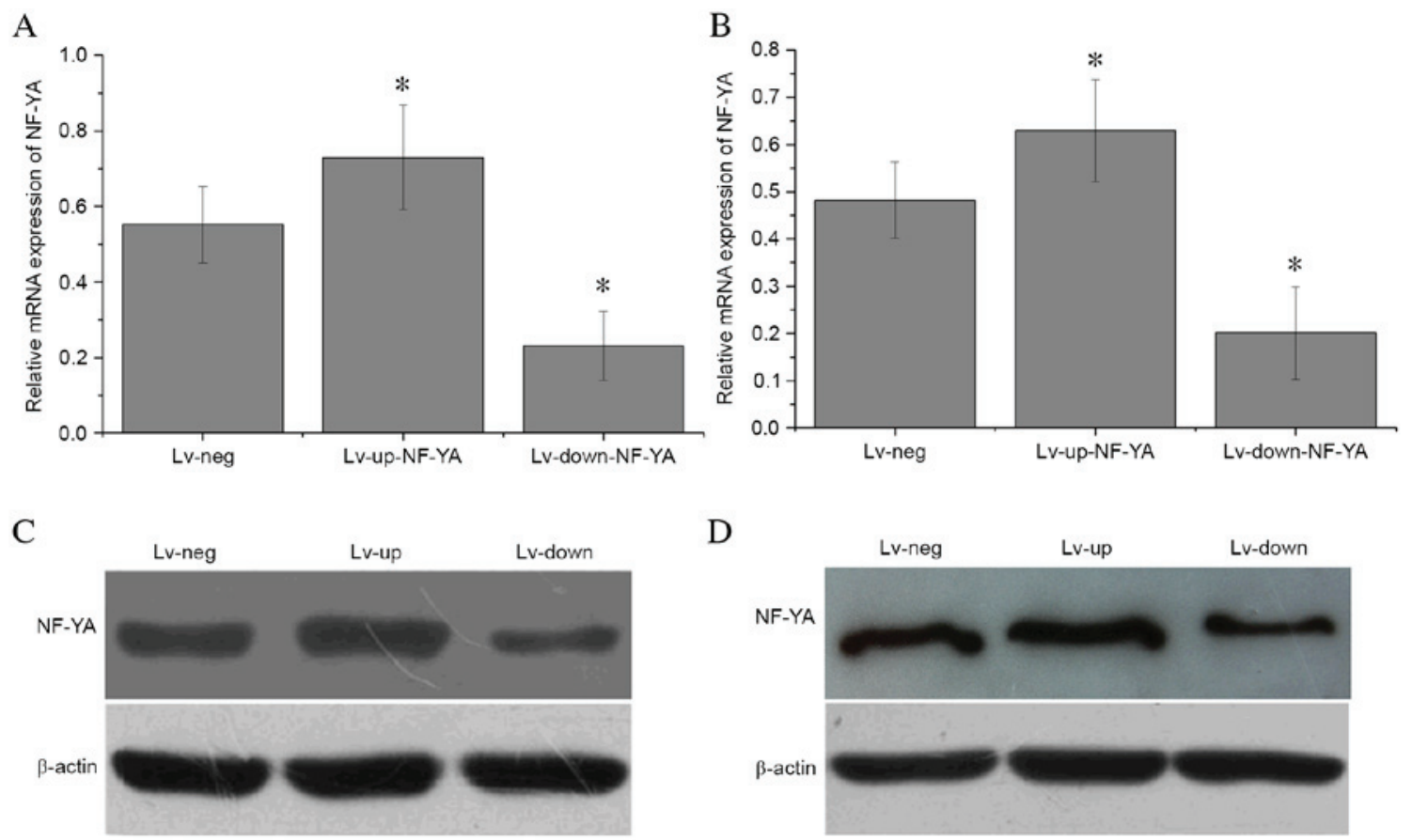

$\mathrm{D}$
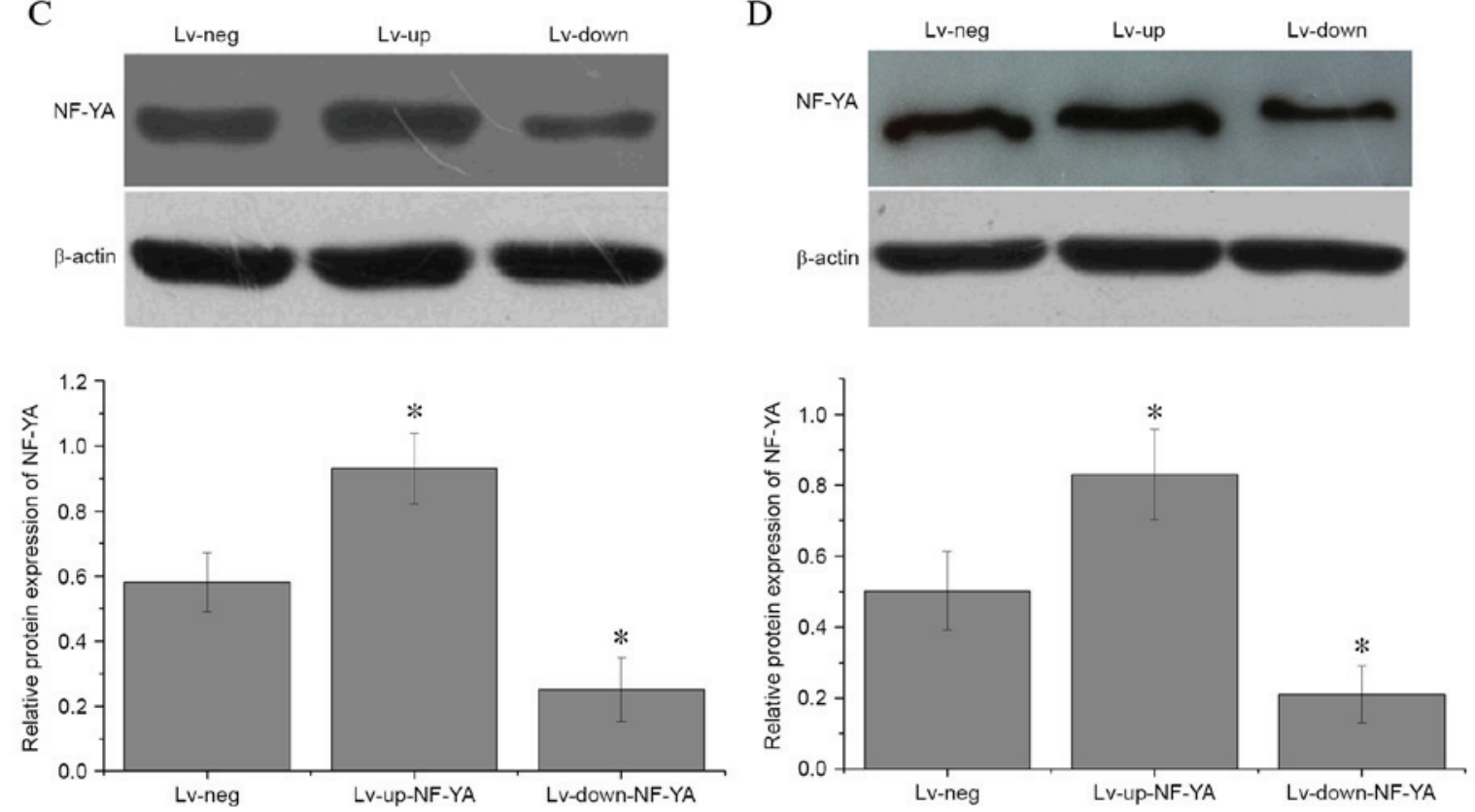

Figure 2. NF-YA mRNA and protein expression levels in OS cell lines following transfection with lentiviral vectors to upregulate and downregulate NF-YA. NF-YA mRNA expression levels in (A) U2-OS and (B) HOS cells were detected by reverse transcription-quantitative polymerase chain reaction relative to $\beta$-actin. NF-YA protein expression levels in (C) U2-OS and (D) HOS cells were detected by western blot and quantified relative to $\beta$-actin. Data are presented as the mean \pm standard deviation of six independent experiments. "P $<0.05$ vs. Lv-neg. NF-YA, alpha subunit of nuclear transcription factor $Y$; Lv-neg, negative control lentivirus vector; Lv-up-NF-YA, lentivirus vector to upregulate NF-YA; Lv-down-NF-YA, lentivirus vector to downregulate NF-YA.

at $4^{\circ} \mathrm{C}$ : mouse anti-NF-YA (1:500; catalog no. sc-17753; Santa Cruz Biotechnology, Inc., Dallas, TX, USA), mouse anti-FASN (1:500; catalog no. sc-55580; Santa Cruz Biotechnology, Inc.) and mouse anti- $\beta$-actin antibody (1:2,000; catalog no. sc-47778; Santa Cruz Biotechnology, Inc.). Following incubation with a goat anti-mouse horseradish peroxidase-conjugated secondary antibody (1:5,000; catalog no. 610-103-043; Rockland Immunochemicals, Inc., Pottstown, PA, USA) for $1.5 \mathrm{~h}$ at room temperature, immunoreactive bands were visualized using an Enhanced Chemiluminescence reagent (Thermo Fisher Scientific, Inc.). The intensity of western blot bands was measured using ImageJ software version 1.48 (National Institutes of Health, Bethesda, MD, USA). Six independent experiments were performed over multiple days.

Migration assays. Cell migration was assessed using a 'wound-healing assay', which measures the ability of cells to migrate into a two-dimensional space in vitro. In brief, cells were cultured to $80 \%$ confluence in 6-well tissue culture dishes to a density of $\sim 5 \times 10^{6}$ cells/well. A line of cells was removed by scraping a line of cells from the center of the plate using a rubber policeman (Thermo Fisher Scientific, Inc.). Cultures were rinsed with PBS and fresh medium alone without $10 \%$ FBS was then added before the cells were incubated at $37^{\circ} \mathrm{C}$ for $24 \mathrm{~h}$. Images were captured at 1 and $24 \mathrm{~h}$ using an electron microscope and camera (Canon, Inc., Tokyo, Japan), and the migrated distance was measured using ImageJ software version 1.48 (National Institutes of Health). For each sample, the cell migration rate was calculated by counting the number of migrated cells in three fields of view per well. A total of six independent experiments were performed on separate days.

Transwell invasion assays. Invasion of OS cells was measured using the BD BioCoat Matrigel Invasion Chamber (BD Biosciences, Franklin Lakes, NJ, USA) according to the manufacturer's protocol. The medium in the lower chamber contained 5\% fetal calf serum (Gibco; Thermo 
A

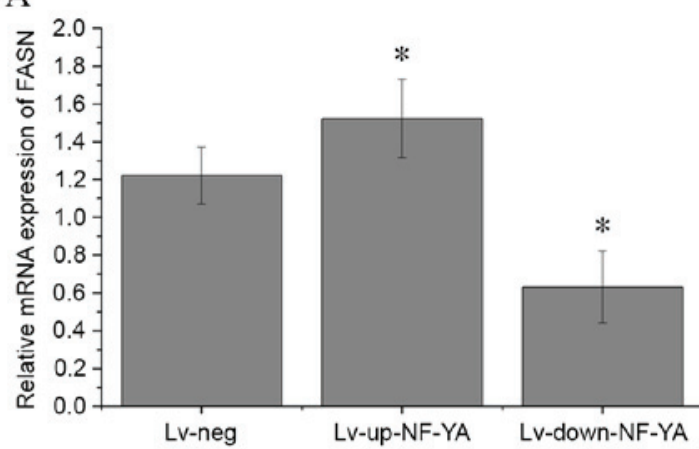

$\mathrm{C}$
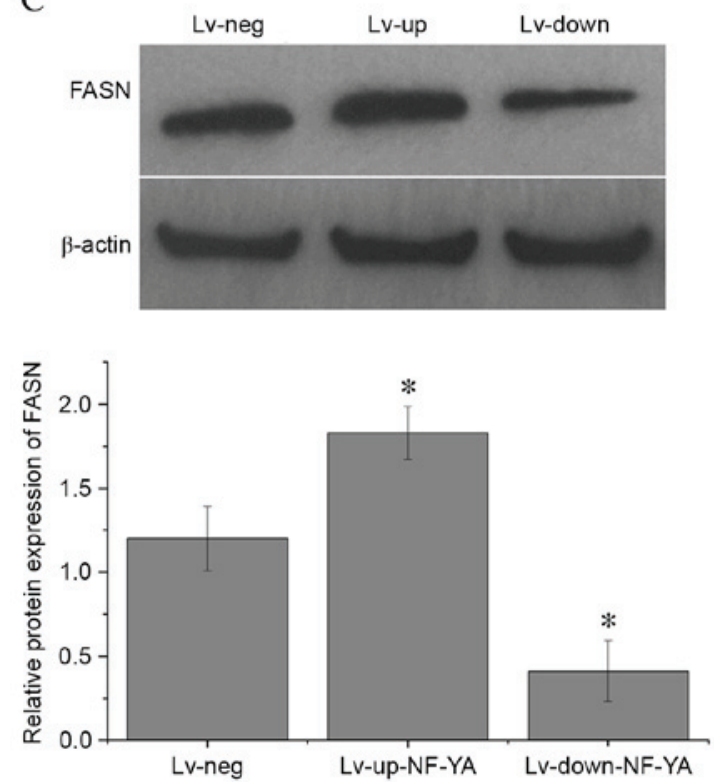

B

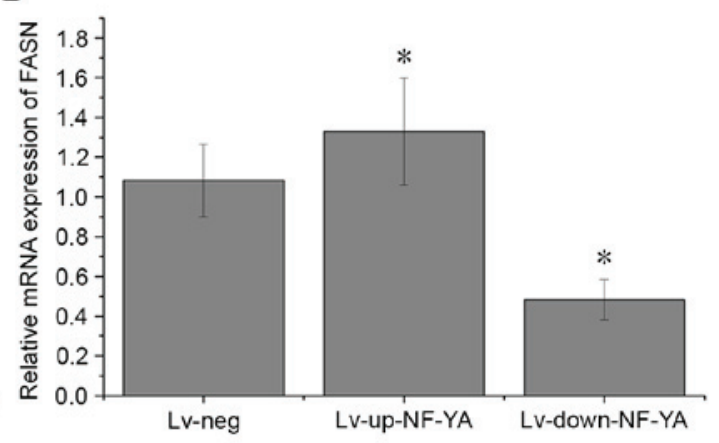

D
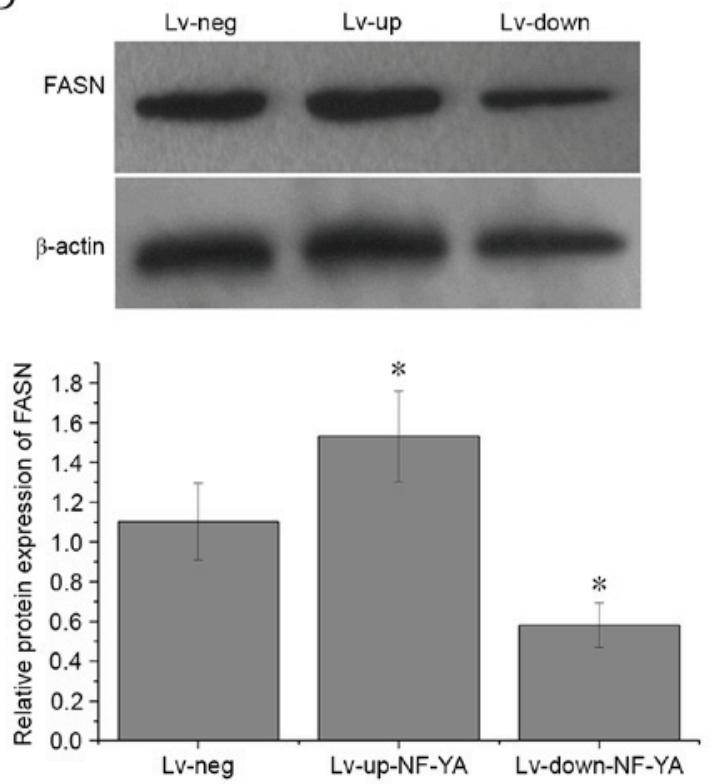

Figure 3. NF-YA regulates FASN expression in OS cell lines following transfection with lentivirus vectors to upregulate and downregulate NF-YA. FASN mRNA expression levels in (A) U2-OS and (B) HOS cells were detected by reverse transcription-quantitative polymerase chain reaction relative to $\beta$-actin. FASN protein expression levels in (C) U2-OS and (D) HOS cells were detected by western blot and quantified relative to $\beta$-actin. Data are presented as the mean \pm standard deviation of six independent experiments. ${ }^{*} \mathrm{P}<0.05$ vs. Lv-neg. NF-YA, alpha subunit of nuclear transcription factor Y; FASN, fatty acid synthase; Lv-neg, negative control lentivirus vector; Lv-up-NF-YA, lentivirus vector to upregulate NF-YA; Lv-down-NF-YA, lentivirus vector to downregulate NF-YA.

Fisher Scientific, Inc.) as a source of chemoattractants. Cells $\left(1 \times 10^{3} /\right.$ well) were suspended in serum-free medium and added to the upper chambers at the same time. Following $24 \mathrm{~h}$ of incubation, cells that passed through the Matrigel-coated membrane were fixed in methanol for $10 \mathrm{~min}$ and stained with Diff-Quik (Sysmex Corporation, Kobe, Japan). Images (original magnification, $\mathrm{x} 400$ ) were captured using an electron microscope and camera (Canon, Inc.). Images of three fields of view per membrane were captured and analyzed using ImageJ software version 1.48 (National Institutes of Health), with six independent experiments performed on separate days.

Statistical analysis. Data are expressed as the mean \pm standard deviation. Student's t-tests were used for two-sample comparisons and one-way analysis of variance was used to compare $>3$ samples. $\mathrm{P}<0.05$ was considered to indicate a statistically significant difference. All analyses were performed using SPSS software (version, 13.0; SPSS, Inc., Chicago, IL, USA).

\section{Results}

Elevated NF-YA expression in OS cells. The mRNA and protein expression levels of NF-YA were assessed in U2-OS, HOS and HOB cells by RT-qPCR and western blot analyses, respectively. Expression levels of NF-YA mRNA (Fig. 1A) and protein (Fig. 1B) were significantly lower in the HOB osteoblast cell line when compared with the U2-OS and HOS OS cell lines, $(\mathrm{P}=0.01$; Fig. 1A). These results suggest that increased NF-YA expression may be a feature of OS cells.

Construction of lentivirus vectors and transfection into OS cells. U2-OS and HOS cells were transfected for $6 \mathrm{~h}$ with lentivirus vectors to upregulate (Lv-up-NF-YA) or downregulate (Lv-down-NF-YA) NF-YA expression. A non-targeting control (Lv-neg) was used as a negative control. NF-YA mRNA (Fig. 2A and B) and protein (Fig. 2C and D) expression levels were then assessed by RT-qPCR and western blot analyses, respectively. NF-YA mRNA expression was 
A

Lv-neg

Lv-up

Lv-down
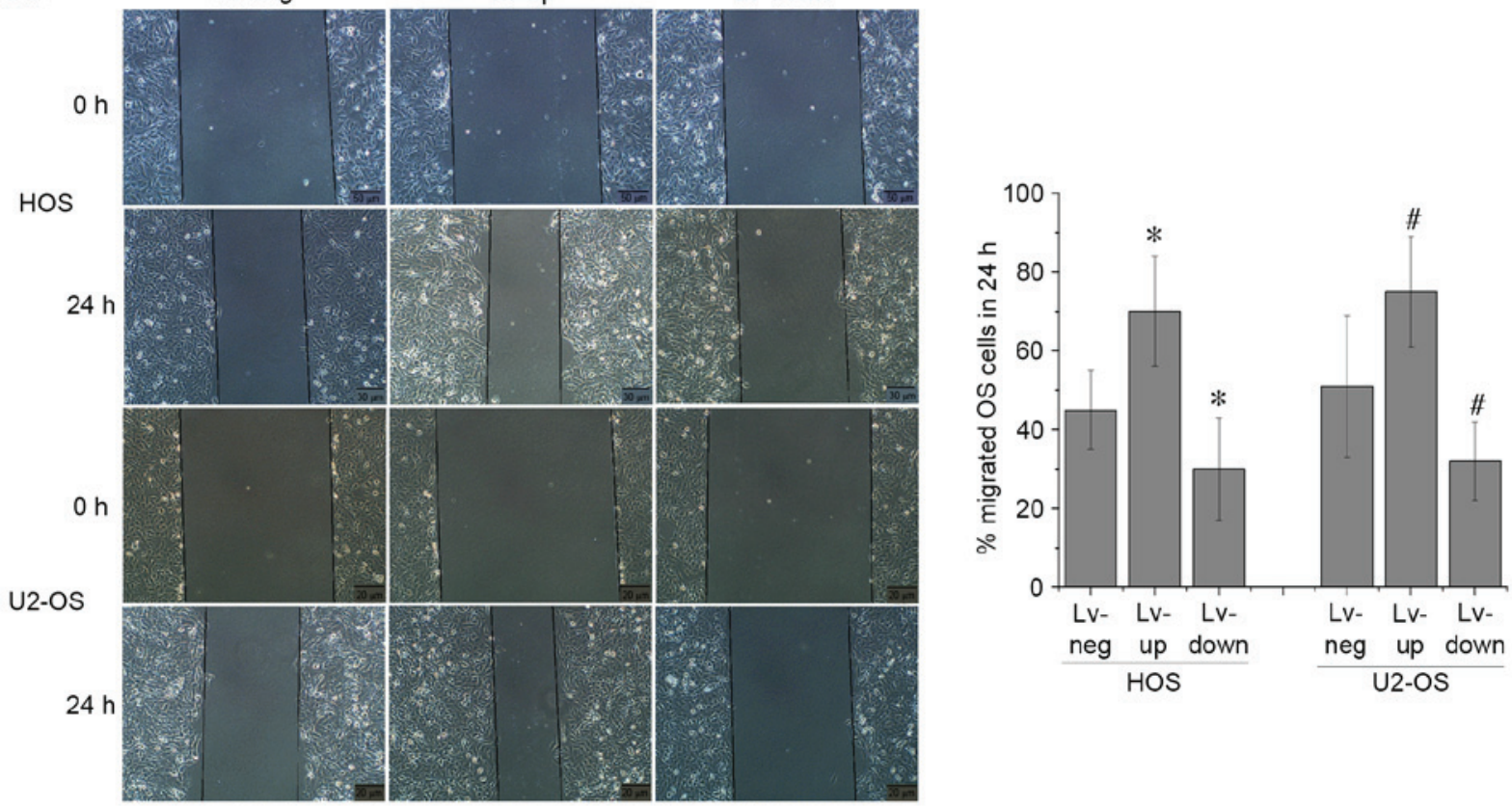

B
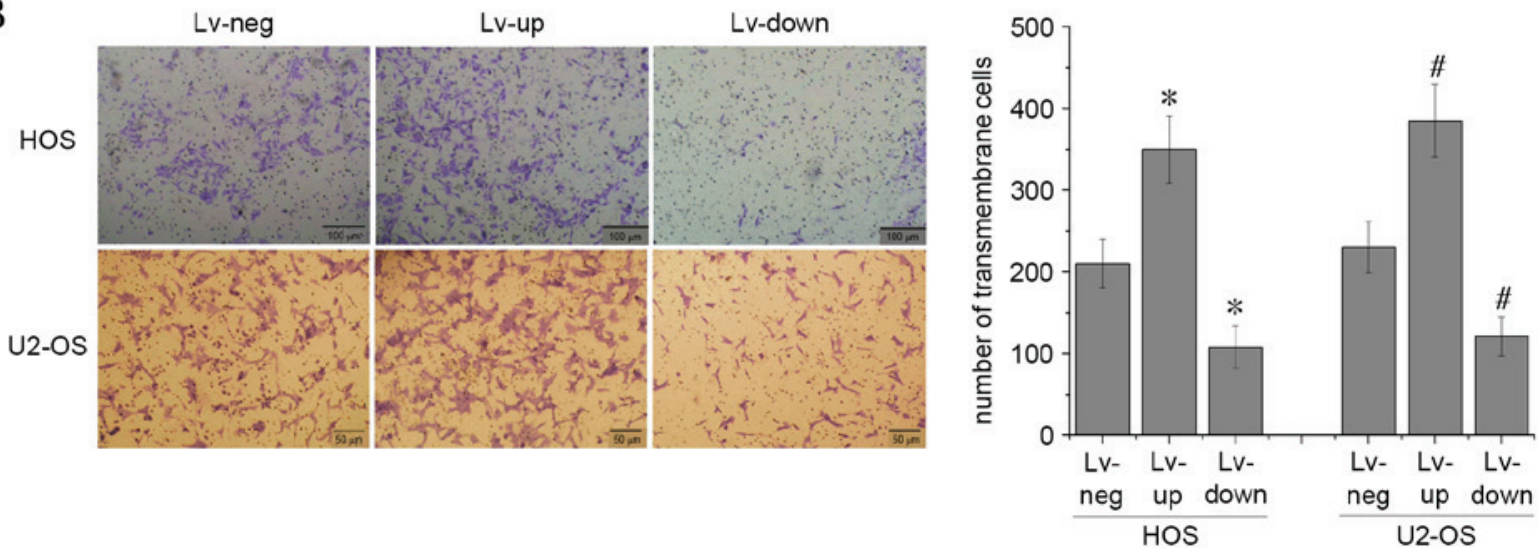

Figure 4. Effect of altered NF-YA expression on the migratory and invasive abilities of OS cells. Following transfection of U2-OS and HOS cells with lentivirus vectors to upregulate and downregulate NF-YA, (A) cell migration was assessed by wound healing assay, and (B) cell invasion was assessed by Transwell assay. Magnification, $\mathrm{x} 400$. Data are presented as the mean \pm standard deviation of six independent experiments. ${ }^{*} \mathrm{P}<0.05$ vs. Lv-neg in $\mathrm{HOS}$ cells; ${ }^{~} \mathrm{P}<0.05 \mathrm{vs}$. Lv-neg in U2-OS cells. NF-YA, alpha subunit of nuclear transcription factor Y; Lv-neg, negative control lentivirus vector; Lv-up, lentivirus vector to upregulate NF-YA; Lv-down, lentivirus vector to downregulate NF-YA

significantly decreased when compared with Lv-neg in U2-OS ( $\mathrm{P}=0.01$; Fig. 2A) and HOS cells ( $\mathrm{P}=0.01$; Fig. 2B) transfected with Lv-down-NF-YA. By contrast, NF-YA expression was significantly enhanced in $\mathrm{U} 2-\mathrm{OS}(\mathrm{P}=0.03$; Fig. 2A) and HOS cells ( $\mathrm{P}=0.01$; Fig. 2B) transfected with Lv-up-NF-YA compared with those transfected with Lv-neg. In addition, the protein expression levels of NF-YA in U2-OS $(\mathrm{P}=0.01 ;$ Fig. 2C) and HOS cells ( $\mathrm{P}=0.01 ;$ Fig. 2D) transfected with Lv-up-NF-YA were significantly increased compared with Lv-neg-transfected cells. Furthermore, NF-YA protein expression was significantly lower in U2-OS $(\mathrm{P}<0.001$; Fig. 2C) and HOS cells ( $\mathrm{P}=0.01$; Fig. 2D) transfected with Lv-down-NF-YA compared with those transfected with Lv-neg. These results indicate that the plasmids targeting NF-YA were constructed and transfected into OS cells successfully.
$N F-Y A$ regulates FASN expression in OS cells. In order to investigate whether NF-YA levels affect FASN expression in OS cells, the mRNA and protein expression levels of FASN were detected in U2-OS and HOS cells transfected with Lv-up-NF-YA, Lv-down-NF-YA and Lv-neg by RT-qPCR and western blot analyses, respectively. FASN mRNA expression was significantly decreased when compared with Lv-neg in $\mathrm{U} 2-\mathrm{OS}(\mathrm{P}=0.02$; Fig. $3 \mathrm{~A})$ and HOS cells $(\mathrm{P}=0.01$; Fig. 3B) transfected with Lv-down-NF-YA. By contrast, FASN mRNA expression was increased in $\mathrm{U} 2-\mathrm{OS}(\mathrm{P}=0.01$; Fig. $3 \mathrm{~A})$ and $\mathrm{HOS}$ cells $(\mathrm{P}<0.001$; Fig. 3B) transfected with Lv-up-NF-YA when compared with those transfected with Lv-neg. In addition, FASN protein expression levels in U2-OS ( $\mathrm{P}=0.01$; Fig. $3 \mathrm{C})$ and HOS cells ( $\mathrm{P}=0.02$; Fig. 3D) transfected with Lv-up-NF-YA was significantly higher than those transfected with Lv-neg. FASN protein levels were significantly decreased in U2-OS 

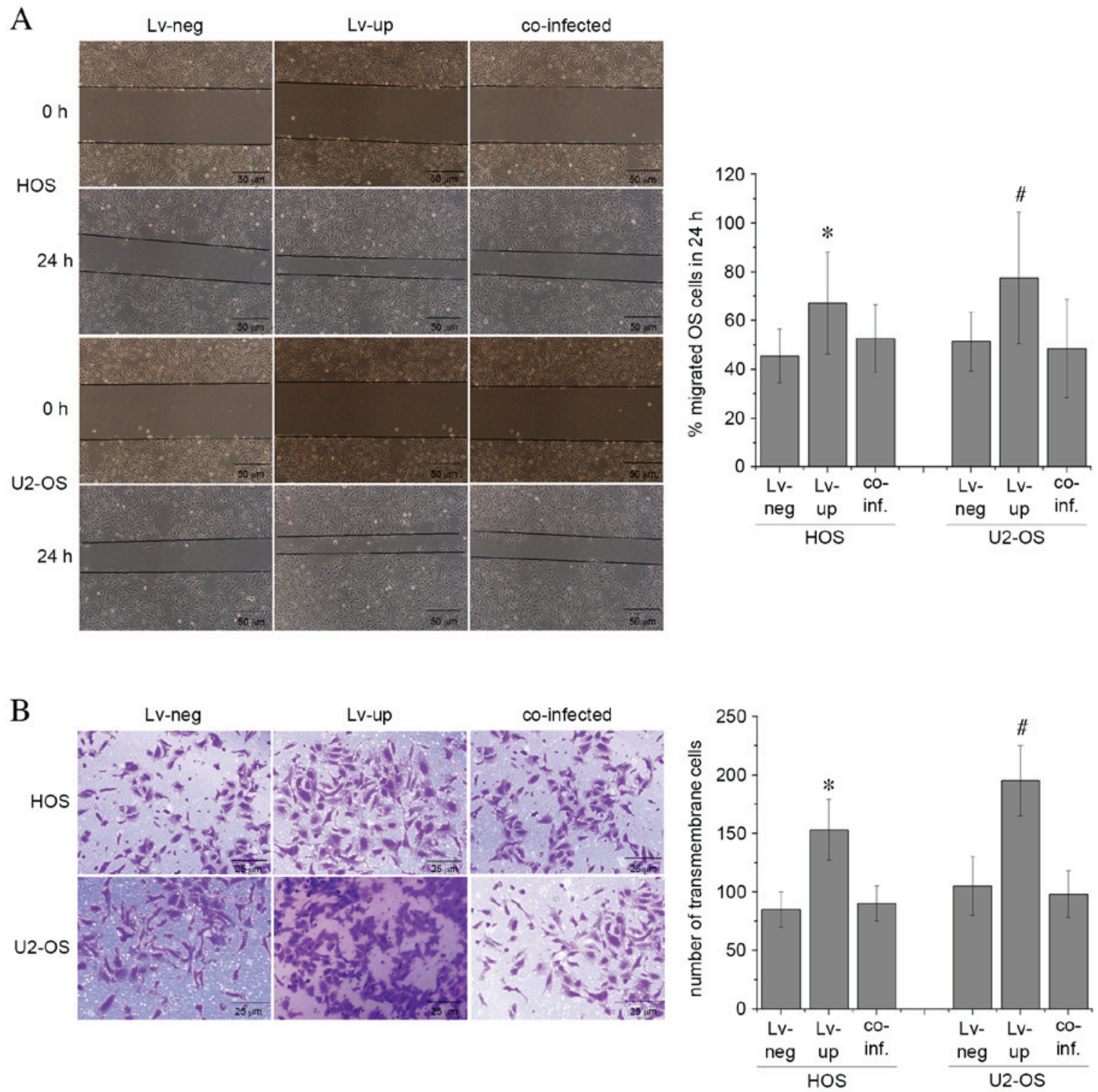

Figure 5. Effect on migratory and invasive abilities of OS cells following transfection of U2-OS and HOS cells with lentivirus vector to upregulate NF-YA, and co-infection with lentivirus vectors to upregulate NF-YA and downregulate FASN. (A) Cell migration was assessed by wound-healing assay, and (B) cell invasion was assessed using a Transwell assay. Magnification, $x 400$. Data are presented as the mean \pm standard deviation of 6 independent experiments. ${ }^{*} \mathrm{P}<0.05$ vs. Lv-neg in HOS cells; ${ }^{*} \mathrm{P}<0.05$ vs. Lv-neg in U2-OS cells. NF-YA, alpha subunit of nuclear transcription factor Y; FASN, fatty acid synthase; Lv-neg, negative control lentivirus vector; Lv-up, lentivirus vectors to upregulate NF-YA; co-infected/co-inf., co-infection with lentivirus vectors to upregulate NF-YA and downregulate FASN.

( $\mathrm{P}=0.01$; Fig. $3 \mathrm{C})$ and HOS cells $(\mathrm{P}=0.01$; Fig. 3D) transfected with Lv-down-NF-YA compared with cells treated with Lv-neg. These findings indicate that alterations in NF-YA expression may affect FASN expression in OS cells.

NF-YA alters the malignant phenotype of OS cells in vitro. In order to investigate the effect of altered NF-YA expression levels on the malignant phenotype of OS cells, cell migration and invasion abilities were assessed by wound healing and Transwell invasion assays, respectively, in U2-OS and HOS cells transfected with Lv-up-NF-YA, Lv-down-NF-YA and Lv-neg. The results revealed that OS cell migration (Fig. 4A) and invasion (Fig. 4B) capabilities were significantly increased in cells with elevated NF-YA levels when compared with cells transfected with Lv-neg. In addition, these markers of a malignant phenotype were significantly inhibited in U2-OS and HOS cells following transfection with Lv-down-NR-YA compared with those transfected with Lv-neg ( $\mathrm{P}=0.01$; Fig. 4). These findings suggest that elevated NF-YA levels may promote a malignant phenotype in OS cells in vitro.

NF-YA alters the malignant phenotype of OS by regulating FASN expression. To investigate whether NF-YA facilitates the malignant phenotype of OS cells by activating FASN, the migration and invasion capabilities of U2-OS and HOS cells infected with Lv-neg,Lv-up-NF-YA, and co-infected with Lv-down-FASN and Lv-up-NF-YA were assessed. The proportion of migrated HOS and U2-OS cells was significantly higher following transfection with LV-up-NF-YA when compared with cells transfected with $L V$-neg $(\mathrm{P}=0.02$ and $\mathrm{P}=0.01$, respectively; Fig. $5 \mathrm{~A})$, with a 
migration rate of $67.2 \pm 21 \%$ in HOS and $77.5 \pm 27 \%$ in U2-OS cells when transfected with Lv-up-NF-YA, compared with $45.5 \pm 11 \%$ of HOS and $51.4 \pm 12 \%$ of U2-OS cells in the LV-neg group. Notably, this effect was abolished in the co-transfected cells, with migration rates of $52.6 \pm 14 \%$ in HOS and $48.5 \pm 20 \%$ in U2-OS cells following co-transfection with Lv-down-FASN and Lv-up-NF-YA. No statistically significant difference in cell migration was observed between co-transfected U2-OS and HOS cells when compared with Lv-neg-transfected cells $(\mathrm{P}=0.07$ and $\mathrm{P}=0.06$, respectively; Fig. 5A). The number of HOS and U2-OS cells that had traversed the membrane was significantly higher when transfected with LV-up-NF-YA compared with LV-neg $(\mathrm{P}=0.01$ and $\mathrm{P}=0.01$, respectively; Fig. $5 \mathrm{~B})$, with $153 \pm 26$ transmembrane HOS cells/high power field, $x 400(\mathrm{Hp})$ and 195 \pm 30 transmembrane U2-OS cells/Hp transfected with Lv-up-NF-YA, compared with $85 \pm 15$ transmembrane HOS cells/Hp and $105 \pm 25$ transmembrane U2-OS cells/Hp in the Lv-neg group. However, in the co-infected group, there were $90 \pm 15$ transmembrane HOS cells/Hp and 98 \pm 20 transmembrane U2-OS cells/Hp, which was not significantly different when compared with HOS and U2-OS cells transfected with Lv-neg $(\mathrm{P}=0.01$ and $\mathrm{P}=0.01$, respectively; Fig. $5 \mathrm{~B})$. These results suggest that the effect of OS tumor cell migration and invasion mediated by NF-YA was reversed by silencing FASN. This indicates that NF-YA may promote the malignant phenotype of OS cells, in part, by regulating FASN expression.

\section{Discussion}

NF-Y is a ubiquitous protein, composed of 3 subunits, NF-YA, NF-YB and NF-YC, whose genes are highly conserved from yeast to mammals (20). While previous studies have aimed to understand the biological role of NF-Y, recent findings have revealed that NF-Y is involved in malignant tumor development (21-23). Clinically, elevated NF-Y genes indicate a poor prognosis in various cancers, such as breast and lung cancers (24). In the present study, expression of NF-YA was higher in OS cells compared with osteoblastic HOB cells. In addition, the malignant phenotype was inhibited in OS cells in vitro when NF-YA expression was silenced, while ectopic NF-YA expression promoted a malignant phenotype. These findings suggest that NF-YA may be involved in mediating the malignant phenotype of OS. However, the cellular and molecular mechanisms of this effect remain unclear.

FASN is an important enzyme for endogenous lipogenesis in mammals and is responsible for catalyzing the synthesis of long-chain fatty acids (25). Previous studies have demonstrated elevated FASN expression in a variety of human tumors, but low expression levels in normal tissues (26-28). Due to this increased expression in malignant tumor tissues, FASN may represent a novel diagnostic marker and therapeutic target. In addition, previous studies have revealed that inhibition of FASN promotes apoptosis, and reduces cell growth and metastasis in vitro and in vivo $(29,30)$. FASN has been previously demonstrated to be overexpressed in OS tissues, while inhibition of FASN suppresses the malignant phenotype by inhibiting the phosphoinositide 3-kinase/Akt serine/threonine kinase 1/nuclear factor kappa $\kappa$-light-chain-enhancer of activated B cells signaling pathway $(17,18)$. However, the molecular signaling pathways resulting from upregulated FASN expression in OS remain to be fully elucidated. NF-Y is an important nuclear transcription factor that regulates the expression of numerous genes (31-33). Previous studies have demonstrated that NF-Y expression is a key component in the regulation of FASN expression by promoter activity in primary hepatocytes (34). In the present study, ectopic NF-YA expression was demonstrated to upregulate FASN expression in OS cells, while knockdown of NF-YA expression resulted in inhibition of FASN expression. In addition, the effect of NF-YA on OS cell migration and invasion was reversed by silencing FASN. These results suggest that NF-YA promotes cell proliferation, invasion and migration in $\mathrm{OS}$, in part, via regulation of FASN expression.

The findings of the present study indicate that increased expression of NF-YA may promote a malignant phenotype in OS cells via modulation of FASN expression. However, further in vivo experiments are necessary to establish whether inhibition of NF-YA may represent a novel strategy in the management of OS.

\section{Acknowledgements}

The present study was supported by grants from The National Natural Science Foundation of China (grant no. 81260400) and The Natural Science Foundation of Jiangxi Province (grant no. 20114BAB205093).

\section{References}

1. Salah S, Ahmad R, Sultan I, Yaser S and Shehadeh A: Osteosarcoma with metastasis at initial diagnosis: Current outcomes and prognostic factors in the context of a comprehensive cancer center. Mol Clin Oncol 2: 811-816, 2014.

2. Lewis VO: What's new in musculoskeletal oncology: J Bone Joint Surg Am 91: 1546-1556, 2009.

3. Iwamoto Y, Tanaka K, Isu K, Kawai A, Tatezaki S, Ishii T, Kushida K, Beppu Y,Usui M,Tateishi A, et al: Multiinstitutional phase II study of neoadjuvant chemotherapy for osteosarcoma (NECO study) in Japan: NECO-93J and NECO-95J. J Orthop Sci 14: 397-404, 2009.

4. Cho Y, Jung GH, Chung SH, Kim JY, Choi Y and Kim JD: Long-term survivals of stage IIb osteosarcoma: A 20-year experience in a single institution. Clin Orthop Surg 3: 48-54, 2011.

5. Bölling T, Schuller P, Distelmaier B, Schuck A, Ernst I, Gosheger G, Winkelmann W, Dirksen U, Jürgens H, Kronholz HL, et al: Perioperative high-dose rate brachytherapy using a bendy applicator (flab): Treatment results of 74 patients. Anticancer Res 28: 3885-3890, 2008.

6. Lourda M, Trougakos IP and Gonos ES: Development of resistance to chemotherapeutic drugs in human osteosarcoma cell lines largely depends on up-regulation of Clusterin/Apolipoprotein J. Int J Cancer 120: 611-622, 2007.

7. Schwartz CL, Gorlick R, Teot L, Krailo M, Chen Z, Goorin A, Grier HE, Bernstein ML and Meyers P; Children's Oncology Group: Multiple drug resistance in osteogenic sarcoma: INT0133 from the Children's Oncology Group. J Clin Oncol 25: 2057-2062, 2007.

8. Chou AJ and Gorlick R: Chemotherapy resistance in osteosarcoma: Current challenges and future directions. Expert Rev Anticancer Ther 6: 1075-1085, 2006.

9. Dean M, Fojo T and Bates S: Tumour stem cells and drug resistance. Nat Rev Cancer 5: 275-284, 2005.

10. Dolfini D, Gatta R and Mantovani R: NF-Y and the transcriptional activation of CCAAT promoters. Crit Rev Biochem Mol Biol 47: 29-49, 2012.

11. Mojsin M, Topalovic V, Marjanovic Vicentic J and Stevanovic M: Transcription factor NF-Y inhibits cell growth and decreases SOX2 expression in human embryonal carcinoma cell line NT2/D1. Biochemistry (Mosc) 80: 202-207, 2015. 
12. Garipov A, Li H, Bitler BG, Thapa RJ, Balachandran S and Zhang R: NF-YA underlies EZH2 upregulation and is essential for proliferation of human epithelial ovarian cancer cells. Mol Cancer Res 11: 360-369, 2013.

13. Bungartz G, Land H, Scadden DT and Emerson SG: NF-Y is necessary for hematopoietic stem cell proliferation and survival. Blood 119: 1380-1389, 2012.

14. Zhang HT, Zhang D, Zha ZG and Hu CD: Transcriptional activation of PRMT5 by NF-Y is required for cell growth and negatively regulated by the $\mathrm{PKC} / \mathrm{c}-\mathrm{Fos}$ signaling in prostate cancer cells. Biochim Biophys Acta 1839: 1330-1340, 2014.

15. Ma H, Yue X, Gao L, Liang X, Yan W, Zhang Z, Shan H, Zhang H, Spear BT and Ma C: ZHX2 enhances the cytotoxicity of chemotherapeutic drugs in liver tumor cells by repressing MDR1 via interfering with NF-YA. Oncotarget 6: 1049-1063, 2015.

16. Shi Z, Chiang CI, Labhart P, Zhao Y, Yang J, Mistretta TA, Henning SJ, Maity SN and Mori-Akiyama Y: Context-specific role of SOX9 in NF-Y mediated gene regulation in colorectal cancer cells. Nucleic Acids Res 43: 6257-6269, 2015.

17. Liu ZL, Wang G, Peng AF, Luo QF, Zhou Y and Huang SH: Fatty acid synthase expression in osteosarcoma and its correlation with pulmonary metastasis. Oncol Lett 4: 878-882, 2012.

18. Liu ZL, Mao JH, Peng AF, Yin QS, Zhou Y, Long XH and Huang SH: Inhibition of fatty acid synthase suppresses osteosarcoma cell invasion and migration via downregulation of the PI3K/Akt signaling pathway in vitro. Mol Med Rep 7: 608-612, 2013.

19. Livak KJ and Schmittgen TD: Analysis of relative gene expression data using real-time quantitative PCR and the 2(-Delta Delta C(T)) Method. Methods 25: 402-408, 2001.

20. Hackenberg D, Wu Y, Voigt A, Adams R, Schramm P and Grimm B: Studies on differential nuclear translocation mechanism and assembly of the three subunits of the Arabidopsis thaliana transcription factor NF-Y. Mol Plant 5: 876-888, 2012.

21. Ying S, Dunnebier T, Si J and Hamann U: Estrogen receptor alpha and nuclear factor Y coordinately regulate the transcription of the SUMO-conjugating UBC9 gene in MCF-7 breast cancer cells. PLoS One 8: e75695, 2013.

22. Belluti S, Basile V, Benatti P, Ferrari E, Marverti G and Imbriano C: Concurrent inhibition of enzymatic activity and NF-Y-mediated transcription of Topoisomerase-II $\alpha$ by bis-DemethoxyCurcumin in cancer cells. Cell Death Dis 4: e756, 2013.

23. Lützner N, De-Castro Arce J and Rösl F: Gene expression of the tumour suppressor LKB1 is mediated by Sp1, NF-Y and FOXO transcription factors. PLoS One 7: e32590, 2012.

24. Yamanaka K, Mizuarai S, Eguchi T, Itadani H, Hirai H and Kotani H: Expression levels of NF-Y target genes changed by CDKN1B correlate with clinical prognosis in multiple cancers. Genomics 94: 219-227, 2009.
25. Uemoto Y, Abe T, Tameoka N, Hasebe H, Inoue K, Nakajima H, Shoji N, Kobayashi M and Kobayashi E: Whole-genome association study for fatty acid composition of oleic acid in Japanese Black cattle. Anim Genet 42: 141-148, 2011.

26. Bian Y, Yu Y, Wang S and Li L: Up-regulation of fatty acid synthase induced by EGFR/ERK activation promotes tumor growth in pancreatic cancer. Biochem Biophys Res Commun 463: 612-617, 2015.

27. Bauerschlag DO, Maass N, Leonhardt P, Verburg FA, Pecks U, Zeppernick F, Morgenroth A, Mottaghy FM, Tolba R, Meinhold-Heerlein I and Bräutigam K: Fatty acid synthase overexpression: Target for therapy and reversal of chemoresistance in ovarian cancer. J Transl Med 13: 146, 2015.

28. Grube S, Dunisch P, Freitag D, Klausnitzer M, Sakr Y, Walter J, Kalff R and Ewald C: Overexpression of fatty acid synthase in human gliomas correlates with the WHO tumor grade and inhibition with Orlistat reduces cell viability and triggers apoptosis. J Neurooncol 118: 277-287, 2014.

29. Agostini M, Almeida LY, Bastos DC, Ortega RM, Moreira FS, Seguin F, Zecchin KG, Raposo HF, Oliveira HC, Amoêdo ND, et al: The fatty acid synthase inhibitor orlistat reduces the growth and metastasis of orthotopic tongue oral squamous cell carcinomas. Mol Cancer Ther 13: 585-595, 2014.

30. Zaytseva YY, Harris JW, Mitov MI, Kim JT, Butterfield DA, Lee EY, Weiss HL, Gao T and Evers BM: Increased expression of fatty acid synthase provides a survival advantage to colorectal cancer cells via upregulation of cellular respiration. Oncotarget 6: 18891-18904, 2015.

31. Xu H, Fu J, Ha SW, Ju D, Zheng J, Li L and Xie Y: The CCAAT box-binding transcription factor NF-Y regulates basal expression of human proteasome genes. Biochim Biophys Acta 1823: 818-825, 2012

32. Lu YH, Dallner OS, Birsoy K, Fayzikhodjaeva G and Friedman JM: Nuclear Factor-Y is an adipogenic factor that regulates leptin gene expression. Mol Metab 4: 392-405, 2015.

33. Hida M, Hamanaka R, Okamoto O, Yamashita K, Sasaki T, Yoshioka $\mathrm{H}$ and Matsuo N: Nuclear factor Y (NF-Y) regulates the proximal promoter activity of the mouse collagen $\alpha 1(\mathrm{XI})$ gene (Col11a1) in chondrocytes. In Vitro Cell Dev Biol Anim 50: 358-366, 2014.

34. Teran-Garcia M, Adamson AW, Yu G, Rufo C, Suchankova G, Dreesen TD, Tekle M, Clarke SD and Gettys TW: Polyunsaturated fatty acid suppression of fatty acid synthase (FASN): Evidence for dietary modulation of NF-Y binding to the Fasn promoter by SREBP-1c. Biochem J 402: 591-600, 2007. 